\title{
GLOBAL ATTRACTOR FOR NAVIER-STOKES EQUATIONS IN CYLINDRICAL DOMAINS
}

Abstract. Global and regular solutions of the Navier-Stokes system in cylindrical domains have already been obtained under the assumption of smallness of (1) the derivative of the velocity field with respect to the variable along the axis of the cylinder, (2) the derivative of force field with respect to the variable along the axis of the cylinder and (3) the projection of the force field on the axis of the cylinder restricted to the part of the boundary perpendicular to the axis of the cylinder. With the same assumptions we prove in this paper the existence of a global attractor for the Navier-Stokes equations and convergence of solutions to the stationary solutions for the large viscosity coefficient.

1. Introduction. We consider the following initial-boundary value problem:

$$
\begin{array}{ll}
v_{, t}+v \cdot \nabla v-\operatorname{div} \mathbb{T}(v, p)=f & \text { in } \Omega \times(0, \infty), \\
\operatorname{div} v=0 & \text { in } \Omega \times(0, \infty), \\
v \cdot n=0 & \text { on } S \times(0, \infty), \\
n \cdot \mathbb{T}(v, p) \cdot \tau_{\alpha}=0, \quad \alpha=1,2, & \text { on } S \times(0, \infty), \\
\left.v\right|_{t=0}=v(0) & \text { in } \Omega .
\end{array}
$$

The domain $\Omega$ is an open and bounded subset of $\mathbb{R}^{3}$ of cylindrical type. The boundary, denoted by $S$, consists of two parts $S_{1}$ and $S_{2}$, where $S_{1}$ is parallel to the axis of the cylinder and $S_{2}$ is perpendicular to that axis. By $n$ and $\tau_{\alpha}$ we denote the unit normal outward vector and unit tangent vectors to $S$. We denote by $v=v(x, t) \in \mathbb{R}^{3}$ the velocity field, by $f=f(x, t) \in \mathbb{R}^{3}$ the external force field, and by $p=p(x, t) \in \mathbb{R}$ the pressure, where $x=$

2000 Mathematics Subject Classification: 34D05, 34D45, 35Q30, 76D03, 76D05.

Key words and phrases: Navier-Stokes equations, global attractor, incompressible fluids, global existence of regular solutions, boundary slip conditions. 
$\left(x_{1}, x_{2}, x_{3}\right)$ is a global Cartesian coordinates system such that the $x_{3}$-axis is the axis of the cylinder. $\mathbb{T}(v, p)$ is the stress tensor equal to $\nu \mathbb{D}(v)-\mathbb{I} p$, where $\mathbb{D}(v)=\nabla v+(\nabla v)^{T}$ denotes the dilatation tensor, $\nu$ is the constant viscosity coefficient and $\mathbb{I}$ is the unit matrix. Moreover, the dot denotes the scalar product in $\mathbb{R}^{3}$.

The existence of regular solutions to the problem (1.1) has been proved in [8], [11], [12], [13] by the Leray-Schauder fixed point theorem under some smallness assumptions on the $L_{2}$-norms of the $x_{3}$-derivatives of the external force and the initial velocity. The next step was to obtain a global in time solution (see e.g. [6]). This is stated in the following theorem which has been proved in [7].

Theorem 1 (global existence). Let $T>0$ be fixed and let $\delta_{k}(T):=\left\|f_{, x_{3}}\right\|_{L_{2}(\Omega \times(k T,(k+1) T))}+\left\|f_{3}\right\|_{L_{2}\left(S_{2} \times(k T,(k+1) T)\right)}+\left\|v_{, x_{3}}(k T)\right\|_{L_{2}(\Omega)}$, where $k \in \mathbb{N}$. Assume that

$$
\begin{aligned}
& f \in L_{\infty}\left(k T,(k+1) T ; L_{6 / 5}(\Omega)\right) \cap L_{2}(\Omega \times(k T,(k+1) T)), \\
& f_{3} \in L_{2}\left(S_{2} \times(k T,(k+1) T)\right), \\
& (\operatorname{rot} f)_{3} \in L_{2}\left(k T,(k+1) T ; L_{6 / 5}(\Omega)\right), \\
& f_{, x_{3}} \in L_{2}(\Omega \times(k T,(k+1) T)) \cap L_{\sigma}(\Omega \times(k T,(k+1) T))
\end{aligned}
$$

and $v(k T) \in H^{1}(\Omega)$. Then there exists a global and regular solution $(v, p)$ to the problem (1.1) such that

$$
\left\|v_{, x_{3}}\right\|_{W_{\sigma}^{2,1}(\Omega \times(k T,(k+1) T))}+\left\|\nabla p_{x_{3}}\right\|_{L_{\sigma}(\Omega \times(k T,(k+1) T))}<A
$$

and

$$
\|v\|_{W_{2}^{2,1}(\Omega \times(k T,(k+1) T))}+\|\nabla p\|_{L_{2}(\Omega \times(k T,(k+1) T))}<c\left(A^{2}+1\right),
$$

with any $\sigma \in(25 / 8,10 / 3)$ and the constant $A$ is chosen for given $T$ independently of $k$ and it satisfies the inequalities

$$
\varphi\left(3 A+D_{k}\right) \delta_{k}(T)+c E_{k} \leq A, \quad c E_{k}<A,
$$

where $\varphi$ is some nonlinear, positive and increasing function, the constant $c$ comes from an imbedding theorem for Sobolev spaces and the constants $D_{k}$ and $E_{k}$ are given by

$$
\begin{aligned}
D_{k}:= & \|f\|_{L_{\infty}\left(k T,(k+1) T ; L_{6 / 5}(\Omega)\right)}+\left\|f_{3}\right\|_{L_{2}\left(S_{2} \times(k T,(k+1) T)\right)}+\|f\|_{L_{2}(\Omega \times(k T,(k+1) T))} \\
& +\left\|(\operatorname{rot} f)_{3}\right\|_{L_{2}\left(k T,(k+1) T ; L_{6 / 5}(\Omega)\right)}+\left\|f_{x_{3}}\right\|_{L_{2}(\Omega \times(k T,(k+1) T))}+d_{1}+d_{2}, \\
E_{k}:= & \left\|f_{x_{3}}\right\|_{L_{\sigma}(\Omega \times(k T,(k+1) T))},
\end{aligned}
$$

where $d_{1}$ and $d_{2}$ come from the energy estimates of weak solutions to the problem (1.1) (see Lemma 2.3). 
In this paper we will show the existence of a global attractor for problem (1.1). We will apply the methods derived in [5] and [11] and use the theory of semiprocesses since in our case the external force $f$ may depend on time.

2. Auxiliary results. We introduce the standard notation that will be frequently used in this paper. Let $\delta>0$ be fixed and

$$
\begin{aligned}
\bar{V}=\left\{v \in \mathcal{C}^{\infty}(\Omega): \operatorname{div} v=0\right. & \text { in } \Omega,\left.v \cdot n\right|_{S}=0 \\
& \text { and } \left.\left(\int_{\Omega}\left|v_{, x_{3}}\right|^{2} d x\right)^{1 / 2}<\delta\right\},
\end{aligned}
$$

and

$$
\begin{aligned}
H & =\text { closure of } \bar{V} \text { in the } L_{2} \text {-norm, } \\
V & =\text { closure of } \bar{V} \text { in the } H^{1} \text {-norm. }
\end{aligned}
$$

We need the space $V_{2}^{k}\left(\Omega^{T}\right)$ defined as follows:

$$
\begin{aligned}
V_{2}^{k}\left(\Omega^{T}\right)=\left\{v:\|v\|_{V_{2}^{k}\left(\Omega^{T}\right)}=\right. & \operatorname{ess~sup}_{t \in(0, T)}\|v\|_{H^{k}(\Omega)} \\
& \left.+\left(\int_{0}^{T}\|\nabla v\|_{H^{k}(\Omega)}^{2} d t\right)^{1 / 2}<\infty\right\}, \quad k \in \mathbb{N} .
\end{aligned}
$$

Now we can define a weak solution to the problem (1.1).

Definition 2.1. By a weak solution to the problem (1.1) we mean a function $v \in V_{2}^{0}\left(\Omega^{T}\right)$ such that $\operatorname{div} v=0,\left.v \cdot n\right|_{S}=0$, satisfying the integral identity

$$
\begin{aligned}
\int_{\Omega^{T}}\left(-v \cdot \varphi_{, t}+\nu \mathbb{D}(v)\right. & \cdot \mathbb{D}(\varphi)+v \cdot \nabla v \cdot \varphi) d x d t \\
& +\left.\int_{\Omega} v \cdot \varphi\right|_{t=T} d x-\left.\int_{\Omega} v \cdot \varphi\right|_{t=0} d x=\int_{\Omega^{T}} f \cdot \varphi d x d t
\end{aligned}
$$

for any $\varphi \in W_{2}^{1,1}\left(\Omega^{T}\right)$.

In order to prove the existence of a weak solution we need the Korn inequality and energy type estimates. The proofs can be found in [12].

Lemma 2.2 (Korn inequality). Assume that $v \in H^{1}(\Omega)$ is such that

$$
\begin{aligned}
& \|\mathbb{D}(v)\|_{L_{2}(\Omega)}^{2}<\infty, \\
& \left.v \cdot n\right|_{S}=0 \\
& \operatorname{div} v=0
\end{aligned}
$$


If $\Omega$ is not axially symmetric, then there exists some constant $c_{1}$ such that

$$
\|v\|_{H^{1}(\Omega)}^{2} \leq c_{1}\|\mathbb{D}(v)\|_{L_{2}(\Omega)}^{2} .
$$

Lemma 2.3 (Energy estimates). Let $T>0$ be given. Let

$$
\begin{aligned}
& a_{1}=\sup _{t}\|f(t)\|_{L_{6 / 5}(\Omega)}, \\
& d_{1}^{2}=\frac{c}{\nu_{1}} a_{1}^{2}+\|v(0)\|_{L_{2}(\Omega)}^{2}, \\
& d_{2}^{2}=\left(\min \left(1, \nu_{2}\right)\right)^{-1} e^{\nu_{1} T}\left(\frac{c}{\nu_{1}} a_{1}^{2}+d_{1}^{2}\right),
\end{aligned}
$$

which do not depend on $k \in \mathbb{N}$ and $\nu / c_{1}=\nu_{1}+\nu_{2}$, where $c_{1}$ is the constant from the Korn inequality (Lemma 2.2). Assume $a_{1}<\infty$ and $v(0) \in L_{2}(\Omega)$. Then

$$
\begin{array}{ll}
\|v(t)\|_{L_{2}(\Omega)} \leq d_{1} & \text { for any } t \geq 0, \\
\|v\|_{V_{2}^{0}(\Omega \times(k T, t))} \leq d_{2} & \text { for } t \in(k T,(k+1) T), k \in \mathbb{N} .
\end{array}
$$

Applying now the Galerkin method and repeating some considerations from $[4$, Ch. 6], we have

Lemma 2.4. Assume $a_{1}<\infty, v(0) \in L_{2}(\Omega)$ and let $T>0$ be given. Then there exists a weak solution to the problem (1.1) in any interval $(k T,(k+1) T)$, $k \in \mathbb{N}$, satisfying

$$
\|v\|_{V_{2}^{0}(\Omega \times(k T,(k+1) T))} \leq d_{2} .
$$

Before we can focus on global attractors, we need two estimates and the uniform Gronwall inequality.

Lemma 2.5. Any solution $v \in H^{2}(\Omega)$ of the elliptic problem

$$
\begin{aligned}
& \operatorname{div} \mathbb{D}(v)=f, \\
& \left.v \cdot n\right|_{S}=0, \\
& \left.n \cdot \mathbb{D}(v) \cdot \tau_{\alpha}\right|_{S}=0, \quad \alpha=1,2,
\end{aligned}
$$

satisfies the estimate

$$
\|v\|_{H^{2}(\Omega)} \leq c\|f\|_{L_{2}(\Omega)} .
$$

Lemma 2.6. Any solution $(v, p) \in H^{2}(\Omega) \times H^{1}(\Omega)$ of the elliptic problem

$$
\begin{aligned}
& \operatorname{div} \mathbb{T}(v, p)=f, \\
& \operatorname{div} v=0, \\
& \left.v \cdot n\right|_{S}=0, \\
& \left.n \cdot \mathbb{T}(v, p) \cdot \tau_{\alpha}\right|_{S}=0, \quad \alpha=1,2,
\end{aligned}
$$

satisfies the estimate

$$
\|v\|_{H^{2}(\Omega)}+\|\nabla p\|_{L_{2}(\Omega)} \leq c\|f\|_{L_{2}(\Omega)} .
$$


Lemma 2.7 (the uniform Gronwall inequality). Let the functions $f, h$, $y:\left[t_{0}, \infty\right) \rightarrow(0, \infty)$ be continuous. Assume that for some $r>0$ and all $t>t_{0}$ we have

$$
y^{\prime}(t) \leq g(t) y(t)+h(t)
$$

and

$$
\int_{t}^{t+r} g(s) d s \leq a_{1}, \quad \int_{t}^{t+r} h(s) d s \leq a_{2}, \quad \int_{t}^{t+r} y(s) d s \leq a_{3} .
$$

Then $y$ satisfies the uniform estimate

$$
y(t+r) \leq\left(\frac{a_{3}}{r}+a_{2}\right) e^{a_{1}} \quad \text { for all } t>t_{0} .
$$

The proofs of Lemmas 2.5 and 2.6 are almost the same as in [1]. We only restrict ourselves to the stationary case. The proof of Lemma 2.7 can be found in [10, Ch. $3, \S 1]$.

3. Existence of a global attractor. In this section we prove the existence of a global attractor to the problem (1.1). We start by recalling some facts and definitions from [3, Ch. 4].

Let us rewrite equation $(1.1)_{1}$ in the abstract form

$$
v_{, t}=A(v, t)=A_{\sigma(t)}(v), \quad t \in \mathbb{R}^{+},
$$

where the right-hand side depends explicitly on the time symbol $\sigma(t)$, which is the collection of all time-dependent coefficients of the equation (in the Navier-Stokes equations that will be the time-dependent external forces). By $\Psi$ we denote some metric or Banach space, which contains the values of $\sigma(t)$ for a.e. $t \in \mathbb{R}_{+}$. Moreover, we assume that $\sigma(t)$, as a function of $t$, belongs to a topological function space $\Xi:=\left\{\xi(\cdot): \xi(t) \in \Psi\right.$ for a.e. $\left.t \in \mathbb{R}_{+}\right\}$.

Replacing the symbol $\sigma(t)$ by the shifted symbol $\sigma(t+h)$ should not change the attractor, hence we introduce a translation invariant subspace $\Sigma \subseteq \Xi$ called the symbol space. Translation invariance means that for all $\sigma \in \Sigma$ the relation $T(h) \sigma(t)=\sigma(t+h) \in \Sigma$ holds, where $T(h): \Xi \rightarrow \Xi$ is the shift operator. In our case, it will be convenient to set $\Sigma=\Sigma\left(\sigma_{0}\right) \equiv$ $\overline{\left\{\sigma_{0}(\cdot+h): h \in \mathbb{R}^{+}\right\}}$, where $\sigma_{0}$ is the time symbol of the initial equation and the closure is taken in the topology of $\Xi$.

Let $v(t)$ be a unique weak and global solution of problem (1.1) with initial data $v_{0}=v(0)$. We define the family of semiprocesses $\left\{U_{\sigma}(t, \tau)\right\}_{t \geq \tau \geq 0}$ acting on $H, U(t, \tau): H \rightarrow H$, by the formula

$$
v(t)=U_{\sigma}(t, \tau) v(\tau),
$$

where $v_{\tau}$ is the initial condition and $\Sigma \ni \sigma(t)=f(\cdot, t)$ is the external force.

By $\mathcal{B}(H)$ we denote the family of all bounded sets of $H$. 
Definition 3.1. A family of processes $\left\{U_{\sigma}(t, \tau)\right\}_{t \geq \tau \geq 0}, \sigma \in \Sigma$, is said to be uniformly bounded if for any $B \in \mathcal{B}(H)$,

$$
\bigcup_{\sigma \in \Sigma} \bigcup_{\tau \in \mathbb{R}^{+}} \bigcup_{t \geq \tau} U_{\sigma}(t, \tau) B \in \mathcal{B}(H) .
$$

Definition 3.2. A set $B_{0} \subset H$ is said to be uniformly absorbing for the family of processes $\left\{U_{\sigma}(t, \tau)\right\}_{t \geq \tau \geq 0}, \sigma \in \Sigma$, if for any $\tau \in \mathbb{R}^{+}$and for every $B \in \mathcal{B}(H)$ there exists $t_{0}=t_{0}(\tau, B)$ such that $\bigcup_{\sigma \in \Sigma} U_{\sigma}(t, \tau) B \subseteq B_{0}$ for all $t \geq t_{0}$. If the set $B_{0}$ is compact, we call the family of processes uniformly compact.

Definition 3.3. A set $P \subset H$ is said to be uniformly attracting for the family of processes $\left\{U_{\sigma}(t, \tau)\right\}_{t \geq \tau \geq 0}, \sigma \in \Sigma$, if for any $\tau \in \mathbb{R}^{+}$,

$$
\lim _{t \rightarrow \infty}\left(\sup _{\sigma \in \Sigma} \operatorname{dist}_{E}\left(U_{\sigma}(t, \tau) B, P\right)\right)=0 .
$$

If the set $P$ is compact, we call the family of processes uniformly asymptotically compact.

Definition 3.4. A closed set $\mathcal{A}_{\Sigma} \subset H$ is said to be a uniform attractor of the family of processes $\left\{U_{\sigma}(t, \tau)\right\}_{t \geq \tau \geq 0}, \sigma \in \Sigma$, if it is uniformly attracting and contained in any closed uniformly attracting set of that family.

The existence of a global attractor is guaranteed by the following theorem:

TheOREM 2. If a family of processes $\left\{U_{\sigma}(t, \tau)\right\}_{t \geq \tau \geq 0}, \sigma \in \Sigma$, is uniformly asymptotically compact, then it has a unique uniform global attractor $\mathcal{A}_{\Sigma}$. The set $\mathcal{A}_{\Sigma}$ is compact in $H$.

The main result in this section reads:

TheOREM 3. There exists a unique global attractor $\mathcal{A}_{\Sigma}$ in $H$ for the family of semiprocesses $\left\{U_{\sigma}(t, \tau)\right\}_{t \geq \tau \geq 0}, \sigma \in \Sigma$, defined by (3.1). The attractor is bounded in $V$, compact and connected in $H$. It attracts bounded sets in $H$.

To prove this theorem we need some estimates.

LEMmA 3.5. There exists a bounded and absorbing set in $H$ for the family of semiprocesses $\left\{U_{\sigma}(t, \tau)\right\}_{t \geq \tau \geq 0}, \sigma \in \Sigma$.

Proof. In view of Lemma 2.3 we see that

$$
\limsup _{t \rightarrow \infty}\|v(t)\|_{L_{2}(\Omega)} \leq d_{1} .
$$

Hence for every $v_{0} \in H$ there exists $t_{0}>0$ such that

$$
v(t) \in B\left(0, \rho_{1}\right) \quad \text { for all } t \geq t_{0},
$$


where $B\left(0, \rho_{1}\right)$ is the ball in $H$ centered at 0 with radius $\rho_{1}>d_{1}$. If $B(0, r) \subset$ $H$ is any ball such that $v_{0} \in B(0, r)$ then there exists $t_{0}=t_{0}(r)$ such that (3.2) holds. This ends the proof.

Lemma 3.6. There exists a bounded and absorbing set in $V$ for the family of semiprocesses $\left\{U_{\sigma}(t, \tau)\right\}_{t \geq \tau \geq 0}, \sigma \in \Sigma$.

Proof. We multiply (1.1) by $\operatorname{div} \mathbb{T}(v, p)$ and integrate over $\Omega$ to obtain

$$
\begin{array}{r}
\underbrace{\int_{\Omega} v_{, t} \cdot \operatorname{div} \mathbb{T}(v, p) d x}_{I_{1}}-\underbrace{\int_{\Omega}|\operatorname{div} \mathbb{T}(v, p)|^{2} d x}_{I_{2}}+\underbrace{\int_{\Omega} v \cdot \nabla v \cdot \operatorname{div} \mathbb{T}(v, p) d x}_{I_{3}} \\
=\int_{\Omega} f \cdot \operatorname{div} \mathbb{T}(v, p) d x .
\end{array}
$$

According to the definition of $\mathbb{T}(v, p)$ we have

$$
I_{1}=\int_{\Omega} v_{, t} \cdot \operatorname{div}(\nu \mathbb{D}(v)-p \mathbb{I}) d x=\underbrace{\int_{\Omega} v_{, t} \cdot \operatorname{div}(\nu \mathbb{D}(v)) d x}_{I_{11}}-\underbrace{\int_{\Omega} v_{, t} \cdot \nabla p d x}_{I_{12}} .
$$

Integrating by parts, we see that $I_{12}$ vanishes due to the boundary conditions. From the Stokes theorem it follows that

$$
\begin{aligned}
I_{11}=\int_{\Omega} \operatorname{div}\left(v_{, t} \cdot \nu \mathbb{D}(v)\right) d x- & \int_{\Omega} \nabla v_{, t} \cdot \nu \mathbb{D}(v) d x \\
& =\int_{S} v_{, t} \cdot \nu \mathbb{D}(v) \cdot n d S-\frac{\nu}{4} \frac{d}{d t} \int_{\Omega}|\mathbb{D}(v)|^{2} d x .
\end{aligned}
$$

The boundary integral vanishes due to the boundary conditions:

$$
\nu \int_{S} v_{, t} \cdot \mathbb{D}(v) \cdot n d S=\nu \int_{S}\left(v_{n, t} \cdot n+v_{\tau_{\alpha}, t} \cdot \tau_{\alpha}\right) \cdot \mathbb{D}(v) \cdot n d S=0 .
$$

Eventually we get

$$
I_{1}=-\frac{\nu}{4} \frac{d}{d t} \int_{\Omega}|\mathbb{D}(v)|^{2} d x
$$

Next we estimate $I_{3}$ by the Hölder and the Minkowski inequalities:

$$
\left|\int_{\Omega} v \cdot \nabla v \cdot \operatorname{div} \mathbb{T}(v, p) d x\right| \leq \nu\|v\|_{L_{6}(\Omega)}\|\nabla v\|_{L_{3}(\Omega)}\|\operatorname{div} \mathbb{T}(v, p)\|_{L_{2}(\Omega)} .
$$

Using the interpolation inequality for $L_{p}$ spaces, the imbedding of $H^{1}$ into $L_{6}$, the Young inequality with $\epsilon$ and Lemma 2.5 yields

$$
\begin{aligned}
& c\|v\|_{L_{6}(\Omega)}\|\nabla v\|_{L_{3}(\Omega)}\|\operatorname{div} \mathbb{T}(v, p)\|_{L_{2}(\Omega)} \\
& \quad \leq c\|v\|_{L_{6}(\Omega)}\|\nabla v\|_{L_{2}(\Omega)}^{1 / 2} \cdot\|\nabla v\|_{L_{6}(\Omega)}^{1 / 2}\|\operatorname{div} \mathbb{T}(v, p)\|_{L_{2}(\Omega)}
\end{aligned}
$$




$$
\begin{aligned}
& \leq c\|v\|_{H^{1}(\Omega)}\|v\|_{H^{1}(\Omega)}^{1 / 2}\|v\|_{H^{2}(\Omega)}^{1 / 2}\|\operatorname{div} \mathbb{T}(v, p)\|_{L_{2}(\Omega)} \\
& \leq c\|v\|_{H^{1}(\Omega)}^{3 / 2}\|\operatorname{div} \mathbb{T}(v, p)\|_{L_{2}(\Omega)}^{3 / 2} \leq c\|v\|_{H^{1}(\Omega)}^{6}+\epsilon\|\operatorname{div} \mathbb{T}(v, p)\|_{L_{2}(\Omega)}^{2}
\end{aligned}
$$

Hence we obtain from (3.3) the following inequality:

$$
\begin{array}{r}
-\frac{\nu}{4} \frac{d}{d t}\|\mathbb{D}(v)\|_{L_{2}(\Omega)}^{2}-\|\operatorname{div} \mathbb{T}(v, p)\|_{L_{2}(\Omega)}^{2}+c\|v\|_{H^{1}(\Omega)}^{6}+\epsilon\|\operatorname{div} \mathbb{T}(v, p)\|_{L_{2}(\Omega)}^{2} \\
\geq \int_{\Omega} f \cdot \operatorname{div} \mathbb{T}(v, p) d x .
\end{array}
$$

Multiplying by $-4 / \nu$, using the Hölder and the Young inequalities, and observing that

$$
\|\operatorname{div} \mathbb{D}(v)\|_{L_{2}(\Omega)}^{2} \leq c\|\operatorname{div} \mathbb{T}(v, p)\|_{L_{2}(\Omega)}^{2},
$$

we get

$$
\frac{d}{d t}\|\mathbb{D}(v)\|_{L_{2}(\Omega)}^{2}+\bar{\nu}\|\operatorname{div} \mathbb{D}(v)\|_{L_{2}(\Omega)}^{2} \leq c\|f\|_{L_{2}(\Omega)}^{2}+c\|v\|_{H^{1}(\Omega)}^{6},
$$

where $\bar{\nu}=4 c / \nu$ and the constant $c$ comes from (3.4). Since

$$
\|\mathbb{D}(v)\|_{L_{2}(\Omega)} \leq c\|\operatorname{div} \mathbb{D}(v)\|_{L_{2}(\Omega)},
$$

we obtain

$$
\frac{d}{d t}\|\mathbb{D}(v)\|_{L_{2}(\Omega)}^{2}+\bar{\nu}\|\mathbb{D}(v)\|_{L_{2}(\Omega)}^{2} \leq c\|f\|_{L_{2}(\Omega)}^{2}+c\|v\|_{H^{1}(\Omega)}^{6} .
$$

In view of (1.2) and by the Sobolev imbedding theorem we get

$$
\int_{k T}^{(k+1) T}\|\mathbb{D}(s)\|^{2} d s \leq A=: a_{3},
$$

Applying Lemma 2.7 (the uniform Gronwall inequality) and next Lemma 2.2 (the Korn inequality) yields

$$
\|v(t)\|_{H^{1}(\Omega)}^{2} \leq \frac{a_{3}}{T}+a_{2} \quad \text { for any } k \geq 1
$$

We see that

$$
v(t) \in B\left(0, \rho_{2}\right) \quad \text { for all } t \geq t_{0},
$$

where $B\left(0, \rho_{2}\right)$ is the ball in $V$ centered at 0 of radius $\rho_{2}>a_{3} / T+a_{2}$. If $B(0, r) \subset H$ is any ball such that $v_{0} \in B(0, r)$ then there exists $t_{0}=t_{0}(r)$ such that (3.5) holds. This ends the proof.

Proof of Theorem 3. We take $\rho=\max \left\{\rho_{1}, \rho_{2}\right\}$. Then due to Lemmas 3.5 and 3.6 there exists an absorbing set $B(0, \rho)$ which is bounded in $V$, and compact in $H$. From Theorem 2 we conclude the proof. 
4. Convergence to stationary solutions for large $\nu$. In this section we will prove the following

TheOREm 4. Let $f$ and $f_{\infty}$ denote the external force fields in the nonstationary and stationary problems respectively. Assume that the viscosity $\nu$ is large compared to the external force field $f_{\infty}$, i.e.

$$
\delta(\nu):=\frac{\nu}{c_{1}}-16 \frac{c_{1}}{\nu^{2}}\left\|f_{\infty}\right\|_{H^{1}(\Omega)}^{4}>0,
$$

If

$$
\left\|f(t)-f_{\infty}\right\|_{L_{6 / 5}(\Omega)} \underset{t \rightarrow \infty}{\longrightarrow} 0,
$$

then the unique solution $v(t)$ of problem (1.1) converges to the unique stationary solution $v_{\infty}$ of problem (1.1), and we have the estimate

$$
\left\|v(t)-v_{\infty}\right\|_{L_{2}(\Omega)}^{2} \leq\left\|v(0)-v_{\infty}\right\|_{L_{2}(\Omega)}^{2} e^{-\delta(\nu) t}+\left\|f-f_{\infty}\right\|_{L_{6 / 5}(\Omega)}^{2}
$$

for $t>0$.

Proof. From [9, Ch. $2, \S 1]$ we know that a stationary solution $v_{\infty}$ exists, it is unique provided $\nu^{2}>c\left\|f_{\infty}\right\|_{L_{2}(\Omega)}$, and

$$
\left\|v_{\infty}\right\|_{H^{1}(\Omega)} \leq \frac{1}{\nu}\left\|f_{\infty}\right\|_{L_{2}(\Omega)} .
$$

Let $V=v(t)-v_{\infty}$. Then $V$ satisfies the system of equations

$$
\begin{array}{ll}
V_{, t}-\operatorname{div} \mathbb{D}(V)=-v \cdot \nabla V-V \cdot \nabla v_{\infty}+f-f_{\infty} & \text { in } \Omega^{T}, \\
\operatorname{div} V=0 & \text { in } \Omega^{T}, \\
V \cdot n=0 & \text { on } S^{T}, \\
n \cdot \mathbb{D}(V) \cdot \tau_{\alpha}=0, \quad \alpha=1,2, & \text { on } S^{T}, \\
\left.V\right|_{t=0}=v(0)-v_{\infty} & \text { in } \Omega .
\end{array}
$$

Multiplying the first equation by $V$, integrating over $\Omega$, and using the Korn inequality (Lemma 2.2) gives

$$
\begin{aligned}
& \frac{1}{2} \frac{d}{d t}\|V\|_{L_{2}(\Omega)}^{2}+\frac{\nu}{c_{1}}\|V\|_{H^{1}(\Omega)}^{2} \\
& \quad \leq 2\|V\|_{L_{4}(\Omega)}\left\|v_{\infty}\right\|_{H^{1}(\Omega)}\|V\|_{L_{4}(\Omega)}+\left\|f-f_{\infty}\right\|_{L_{6 / 5}(\Omega)}^{2} .
\end{aligned}
$$

The first term on the right hand side is estimated as follows:

$4\|V\|_{L_{2}(\Omega)}^{1 / 2}\|\nabla V\|_{L_{2}(\Omega)}^{3 / 2}\left\|v_{\infty}\right\|_{H^{1}(\Omega)} \leq \frac{\nu}{2 c_{1}}\|V\|_{H^{1}(\Omega)}^{2}+8 \frac{c_{1}}{\nu}\|V\|_{L_{2}(\Omega)}^{2}\left\|v_{\infty}\right\|_{H^{1}(\Omega)}^{4}$.

Hence we obtain from (4.3)

$$
\frac{d}{d t}\|V\|_{L_{2}(\Omega)}^{2}+\frac{\nu}{c_{1}}\|V\|_{L_{2}(\Omega)}^{2}-16 \frac{c_{1}}{\nu}\|V\|_{L_{2}(\Omega)}^{2}\left\|v_{\infty}\right\|_{H^{1}(\Omega)}^{4} \leq\left\|f-f_{\infty}\right\|_{L_{6 / 5}(\Omega)}^{2} .
$$


Using (4.2), multiplying by $\exp (\delta(\nu) t)$ (see (4.1)) and integrating over $t \geq 0$ gives

$$
\|V(t)\|_{L_{2}(\Omega)}^{2} e^{\delta(\nu) t} \leq\|V(0)\|_{L_{2}(\Omega)}^{2}+\left\|f-f_{\infty}\right\|_{L_{6 / 5}(\Omega)}^{2} e^{\delta(\nu) t},
$$

or equivalently

$$
\|V(t)\|_{L_{2}(\Omega)}^{2} \leq\|V(0)\|_{L_{2}(\Omega)}^{2} e^{-\delta(\nu) t}+\left\|f-f_{\infty}\right\|_{L_{6 / 5}(\Omega)}^{2} .
$$

Choosing $\nu$ large enough so that $\delta(\nu)>0$, we conclude the proof.

5. Regularity of the attractor. In Section 3 we have proved that there exists a bounded, compact and absorbing set $B$ in $H$. Now we will show that this set is in fact compact in $V$. It suffices to bound this set in $H^{2}(\Omega)$.

Lemma 5.1. Assume that $f_{, t} \in L_{2}\left(k T,(k+1) T ; L_{6 / 5}(\Omega)\right) \cap L_{\infty}(k T$, $\left.(k+1) T ; L_{2}(\Omega)\right)$. Then for the family of semiprocessess $\left\{U_{\sigma}(t, \tau)\right\}_{t \geq \tau \geq 0}$, $\sigma \in \Sigma$ there exists a bounded and absorbing set in $H^{2}(\Omega)$.

Proof. First we differentiate $(1.1)_{1}$ with respect to time and take the inner product with $v_{t}$ so that

$$
\begin{aligned}
\frac{1}{2} \frac{d}{d t} \| v_{, t} & \left\|_{L_{2}(\Omega)}^{2}+\frac{\nu}{c_{1}}\right\| v_{, t} \|_{H^{1}(\Omega)}^{2} \\
& \leq 2\|v\|_{H^{1}(\Omega)}\left\|v_{, t}\right\|_{L_{2}(\Omega)}^{1 / 2}\left\|v_{, t}\right\|_{H^{1}(\Omega)}^{3 / 2}+\left\|v_{, t}\right\|_{H^{1}(\Omega)}\left\|f_{, t}\right\|_{L_{6 / 5}(\Omega)} \\
& \leq \epsilon\left\|v_{, t}\right\|_{H^{1}(\Omega)}^{2}+c(1 / \epsilon)\left\|v_{, t}\right\|_{L_{2}(\Omega)}^{2}\|v\|_{H^{1}(\Omega)}^{4}+c(1 / \epsilon)\left\|f_{, t}\right\|_{L_{6 / 5}(\Omega)}^{2} .
\end{aligned}
$$

In view of (1.2) and using the uniform Gronwall inequality (Lemma 2.7), we get

$$
\left\|v_{, t}(t)\right\|_{L_{2}(\Omega)}^{2} \leq\left(\frac{b_{3}}{T}+b_{2}\right) e^{b_{1}}, \quad t \geq T
$$

where

$$
\begin{aligned}
& c \int_{k T}^{(k+1) T}\|v(s)\|_{H^{1}(\Omega)}^{4}(s) d s \leq c A^{2}=: b_{1}, \\
& c \int_{k T}^{(k+1) T}\left\|f_{, t}\right\|_{L_{6 / 5}(\Omega)}^{2}(s) d s \leq b_{2}, \\
& \int_{k T}^{(k+1) T}\left\|v_{, t}(t)\right\|_{L_{2}(\Omega)}^{2}(s) d s \leq A^{2}=: b_{3} .
\end{aligned}
$$


Now we multiply $(1.1)_{1}$ by $\operatorname{div} \mathbb{T}(v, p)$, integrate over $\Omega$ and use the Hölder inequality, so that

$$
\begin{aligned}
& \|\operatorname{div} \mathbb{T}(v, p)\|_{L_{2}(\Omega)}^{2} \leq\left\|v_{, t}\right\|_{L_{2}(\Omega)}\|\operatorname{div} \mathbb{T}(v, p)\|_{L_{2}(\Omega)} \\
& \quad+\|v\|_{\left.L_{6}(\Omega)\right)}\|\nabla v\|_{L_{3}(\Omega)}\|\operatorname{div} \mathbb{T}(v, p)\|_{L_{2}(\Omega)}+\|\operatorname{div} \mathbb{T}(v, p)\|_{L_{2}(\Omega)}\|f\|_{L_{2}(\Omega)} .
\end{aligned}
$$

Applying the Young inequality and repeating the calculation for $I_{3}$ in (3.3), we obtain

$\|\operatorname{div} \mathbb{T}(v, p)\|_{L_{2}(\Omega)}^{2} \leq c\left\|v v_{, t}\right\|_{L_{2}(\Omega)}^{2}+c\|v\|_{H^{1}(\Omega)}^{6}+\epsilon\|\operatorname{div} \mathbb{T}(v, p)\|_{L_{2}(\Omega)}^{2}+c\|f\|_{L_{2}(\Omega)}^{2}$.

In view of $(1.2),(3.4),(5.1)$ and the Korn inequality (Lemma 2.2) we conclude that

$$
\nu\|v(t)\|_{H^{2}(\Omega)}<\infty \quad \text { for almost all } t>T .
$$

Hence there exists a ball $\mathcal{B}\left(0, \rho_{3}\right) \subset H^{2}(\Omega)$ centered at 0 with sufficiently large radius $\rho_{3}$ so that $v(t) \in \mathcal{B}\left(0, \rho_{3}\right)$ for almost all $t>t_{0}=t_{0}\left(v_{0}\right)$.

In view of Theorem 2 and considerations from Section 3 there exists a global attractor in $V$. Thus, we have proved the following

THEOREM 5. There exists a unique global attractor $\mathcal{A}$ in $V$ for the family of semiprocessess $\left\{U_{\sigma}(t, \tau)\right\}_{t \geq \tau \geq 0}$ defined by (3.1). The attractor is bounded in $H^{2}(\Omega)$, compact and connected in $V$. It attracts bounded sets in $V$.

Acknowledgements. Research of W. M. Zajączkowski is partially supported by MNiSW Grant No 1 PO3A 02130 and EC FP6 Marie Curie ToK Programme SPADE2.

\section{References}

[1] W. Alame, On existence of solutions for the nonstationary Stokes system with boundary slip conditions, Appl. Math. (Warsaw) 32 (2005), 195-223.

[2] O. V. Besov, V. P. Il'in and S. M. Nikol'skiı̌, Integral Representations of Functions and Imbedding Theorems, Nauka, Moscow, 1975 (in Russian).

[3] V. V. Chepyzhov and M. I. Vishik, Attractors for Equations of Mathematical Physics, Amer. Math. Soc., Providence, RI, 2001.

[4] O. A. Ladyzhenskaya, Mathematical Theory of Viscous Incompressible Fluid, Nauka, Moscow, 1970 (in Russian).

[5] G. Łukaszewicz, Long time behavior of $2 D$ micropolar fluid flows, Math. Comput. Modelling 34 (2001) 487-509.

[6] P. B. Mucha, Stability of nontrivial solutions of the Navier-Stokes system on the three-dimensional torus, J. Differential Equations 172 (2001), 359-375.

[7] B. Nowakowski and W. M. Zajączkowski, Global existence of solutions to NavierStokes equations in cylindrical domains, Appl. Math. (Warsaw) 36 (2009), 169-182.

[8] J. Rencławowicz and W. M. Zajączkowski, Large time regular solutions to NavierStokes equations in cylindrical domains, Topol. Methods Nonlinear Anal. 32 (2008), 69-87.

[9] R. Temam, Navier-Stokes Equations, North-Holland, 1979. 
[10] R. Temam, Infinite Dimensional Dynamical Systems in Mechanics and Physics, 2nd ed., Springer, New York, 1997.

[11] M. Wiegner and W. M. Zajączkowski, On stability of axially symmetric solutions to Navier-Stokes equations in a cylindrical domain and with boundary slip conditions, in: Banach Center Publ. 70, Inst. Math., Polish Acad. Sci., 2005, 251-278.

[12] W. M. Zajączkowski, Long time existence of regular solutions to Navier-Stokes equations in cylindrical domains under boundary slip conditions, Studia Math. 169 (2005), 243-285.

[13] - Global special regular solutions to the Navier-Stokes equations in a cylindrical domain without the axis of symmetry, Topol. Methods Nonlinear Anal. 24 (2004), 69-105.

Bernard Nowakowski

Institute of Mathematics

Polish Academy of Sciences

Śniadeckich 8

00-956 Warszawa, Poland

E-mail: bernard@impan.pl
Wojciech M. Zajączkowski Institute of Mathematics Polish Academy of Sciences Śniadeckich 8 00-956 Warszawa, Poland E-mail: wz@impan.pl and Institute of Mathematics and Cryptology Military University of Technology Kaliskiego 2 00-908 Warszawa, Poland

Received on 27.6.2007; 